above equations can be obtained from his co-ordinates. We have been able to derive the above equations from the structure of Gibbs and have in this way obtained values of $\varepsilon_{11}$ and $\varepsilon_{14}$ which agree completely with the observed values. The piezo-electric constant $\varepsilon_{14}$ of $\beta$-quartz and the variation of $\varepsilon_{11}$ and $\varepsilon_{14}$ with temperature in the case of $\alpha$-quartz has also been calculated.

$\alpha$-Quartz is a crystal with covalent bindings. The electric moment may therefore be supposed to arise from the displacements of the silicon and oxygen atoms in close neighbourhood. As each silicon is linked to four oxygens and each oxygen to two silicons, six $\mathrm{Si}-\mathrm{O}$ bonds-there are twelve $\mathrm{Si}-\mathrm{O}$ bonds in the unit cell-will correspond to a charge $k_{1} e$ on each atom and the remaining six to a charge $k_{2} e$. In $\beta$-quartz, $k_{1}$ is equal to $k_{2}$, since all the bonds are equivalent.

It can be shown that in a general elastic deformation, the changes in the length of a line after strain, and the changes in the angles between two lines after strain, are completely given in terms of the strain components $u_{x x}, u_{x y}$, etc., and are independent of the rotation components $\omega_{x}, \omega_{y}, \omega_{z}$ (vide Love ${ }^{5}$ ). Any change $\Delta r$ in the length of an $\mathrm{Si}-\mathrm{O}$ bond would produce a moment $k e \Delta r$ in that direction, but the direction cosines of the line after strain are not known, since they involve the rotation com. ponents $\omega_{x}, \omega_{y}, \omega_{z}$. We therefore take three points $P, Q, R$, such that $P$ and $R$ are silicon atoms and $Q$ an oxygen atom, or vice versa, and resolve the electric moments due to the displacement of atoms in the plane of the $\mathrm{Si}-\mathrm{O}$ bonds, $P Q R$ and perpendicular to this plane.

Since the components of strain $u_{x x}$, etc., are very small quantities, it is easy to show that the moments in the plane $P Q R$ may be regarded as produced by a change in the bond directions before strain, and the moment normal to the plane may be taken as half the value of the moment normal to the plane when the displacements of the atoms $P, Q, R$ are entirely normal to the plane. This would be justifiable, since in an elastic deformation of the unit cell, the moments due to $36 \mathrm{O}-\mathrm{Si}-\mathrm{O}$ planes and $6 \mathrm{Si}-\mathrm{O}-\mathrm{Si}$ planes have to be considered.

If $l, m, n$ are the direction cosines of $\mathrm{Si}-\mathrm{O}$ bonds, and $p, q, r$ of the normals to the various planes, we get in summing over all these planes:

$$
\begin{aligned}
p_{x}= & \frac{e}{v}[\Sigma \stackrel{12 \mathrm{Si}-0 \text { bonds }}{k} \cdot \Delta r \cdot l]+ \\
& \frac{e}{8 v}\left[\Sigma \left\{\frac{k_{1}+k_{2}}{2}\left(\Delta r_{1}-\Delta r_{2}\right) \cot \alpha+\right.\right. \\
& \left.\left.\frac{k_{1}-k_{2}}{2} \frac{r_{3} \Delta r_{3}-r\left(\Delta r_{1}+\Delta r_{2}\right)(1-\cos 2 \alpha)}{r \sin 2 \alpha}\right\} p\right]
\end{aligned}
$$

with similar expressions for $p_{y}$ and $p_{z}$; here $P R=r_{3}$; $\Delta r_{1}, \Delta r_{2}, \Delta r_{3}$ are the variations of $P Q, Q R$ and $P R$; $r=P Q=Q R ; \quad \angle P Q R=2 \alpha ; \quad v=$ the volume of the unit cell.

These expressions resolve into equations (1) by giving $p, q, r$ suitable signs. The calculation shows that the moments in the plane of the $\mathrm{Si}-\mathrm{O}$ bonds (the first part of the above expression) make no contribution to the transverse coefficient $\varepsilon_{14}$. Assuming that the moments normal to the plane of the bonds give no contribution to the longitudinal coefficient $\varepsilon_{11}$, we find that if $\varepsilon_{11}=5.1 \times 10^{4}$ we get $k_{1}=0.752$, $k_{2}=0.695$, and $\varepsilon_{14}=1.23 \times 10^{4}$, in complete agreement of the observed values.
A similar calculation when applied to $\beta$-quartz gives $\varepsilon_{11}=0$, and $\varepsilon_{14}=1.07 \times 10^{4}$ for a non-ionic crystal $(k=0.724)$ and $1.47 \times 10^{4}$ for an ionic crystal $(k=1)$. If the moments perpendicular to the plane of the bonds are supposed to give no contribution to the longitudinal coefficient, we find $k_{1}=k_{2}$, which is the case in $\beta$-quartz. The assumption is therefore justified.

The variation of $\varepsilon_{11}$ with temperature in $\alpha$-quartz can be completely explained on the basis of change of co-ordinates with temperature. It is found that at $558^{\circ} \mathrm{C}$. the silicon atoms in $\alpha$-quartz occupy the same positions as they do in $\beta$-quartz. As the transition temperature is reached, the longitudinal coefficient in $\alpha$-quartz drops to zero, but the transverse coefficient changes only by 12 per cent.

Bishambhar Dayal Saksena

Department of Physics,

University of Allahabad. Sept. 3.

${ }^{1}$ Curie, C.R. Acad. Sci., Paris, 95, 914 (1882).

${ }^{2}$ Riecke, Abh. Ges. Wis. Gött., 38, 530 (1892).

${ }^{3}$ Kelvin, Phit. Mag., 36, 331 (1893).

" Gibbs, Proc. Roy. Soc., 110, 443 (1926).

"Love, "Math. Theory of Elasticity", 38 and 39.

\section{Max Planck}

Is a broadcast talk on Max Planck, in which whole-hearted tribute was paid to his great work, I used the words, "If I hesitate to put Planck on a level with Newton and Einstein it must be partly on the ground that he did not seem to know quite what he had done when he did it . . . Planck seems to have been reluctant to realize the range of his innovation." I was, of course, basing my opinion upon his published work. I have just received a letter from Frau Planck, his widow, who has read the talk as printed in the Listener of November 6, 1947, p. 811 , in which she says, "You have done justice to the scientist and to the man in every respect. I should only like to make one small exception. You think that he did not realize the scope of his discovery. Yet he did do so-though of course not in all its consequences. He said to our son with whom he had gone through a book on Galileo, Newton and Kepler, 'Look, my boy, I shall one day be in a book like this'. And to our other son he spoke as Sommerfeld has set it down." The reference is to an article of Prof. Sommerfeld's (Die Neue Zeitung, November 6, 1947), which I have received both from her and from Sommerfeld, where the following passage occurs : "Planck realized the scope of his discovery from the beginning. This is clear from an anecdote which Professor Bavinck, who died recently, repeated to me on the occasion of his lecture at Munich this year : he had it from his friend Erwin Planck, the son of the great man. The father said to the son in 1900, on the occasion of a walk in the Grünewald, 'Today I have made a discovery which is as important as Newton's discovery"."

Naturally both these conversations were unknown to me and do not affect the point that there is nothing in his writings to indicate this early realization of the importance of his discovery. They seem to be of sufficient general interest for the historian of science to warrant me laying them before the readers of Nature.

$$
\text { E. N. DA C. ANDrade }
$$

Department of Physics,

University College, London, W.C.1. 